\title{
Delayed intraorbital infection after craniofacial bone surgery
}

\author{
Joo Sung Jung, \\ Nam Kyu Lim, \\ Dong Hee Kang \\ Department of Plastic and \\ Reconstructive Surgery, Dankook \\ University Hospital, Cheonan, Korea
}

Intraorbital infection shows a low incidence, but it might cause blindness or even death. This case is unusual in that its origin from a craniofacial bone fracture prior to infection of the maxillary sinus. A 33-year-old female patient was referred for right cheek swelling. When she visited the emergency room, we removed right cheek hematoma and bacterial examination was done. In the past, she had craniofacial bone surgical history due to a traffic accident 6 years ago. Next day, the swelling had remained with proptosis and pus was recognized in the conjunctiva. We planned an emergency operation and removed the pus which was already spread inside the orbit. And the evaluation for sinusitis was consulted to the otorhinolaryngology department simultaneously. There were Prevotella oralis and methicillin-resistant Staphylococcus epidermidis bacterial infection in the intraorbital and sinus respectively. Afterwards, the vigorous dressing was done for over a month with intravenous antibiotics. Though the intraorbital infection was resolved, blindness and extraocular movement limitation were inevitable. In conclusion, close follow up of the maxillary sinus in facial bone fracture patients is important and aggressive treatment is needed when an infection is diagnosed.

Keywords: Eye infection / Facial bones / Sinusitis

\section{INTRODUCTION}

Orbital cellulitis is an acute infectious inflammation of the orbit. Sometimes, it could be diffuse with a collection of pus in the orbital cavity. This type of intraorbital infection showed relatively low incidence, but it might extend to the brain, cause blindness or even death [1]. Its most common source is paranasal sinusitis. Among the intraorbital infection patients, twothirds of adults and up to $90 \%$ of children show this cause. Also, the orbital trauma or foreign body in the periorbital region is the second most common cause [2]. We report a rare and serious case of a patient with intraorbital infection who has under-

Correspondence: Nam Kyu Lim

Department of Plastic and Reconstructive Surgery, Dankook University Hospital, 201 Manghyang-ro, Dongnam-gu, Cheonan 31116, Korea

E-mail: linakyer@daum.net

Received July 8, 2019 / Revised August 8, 2019 / Accepted September 9, 2019 gone the craniofacial bone surgery in the past and review of the literature as the intraorbital infection.

\section{CASE REPORT}

A 33-year-old female patient with no particular underlying disease was referred for right cheek swelling 4 days after blunt trauma on the face. When she visited the emergency room, there was hematoma on the right cheek and the patient was unable to open the right eye due to severe swelling. We removed the hematoma on the cheek and bacterial examination was done. She was also consulted to an ophthalmologist but there were limitations in examination due to excessive periorbital swelling.

Six years ago, she was operated for fractured craniofacial bone which the right side fronto-orbital, zygomaticomaxillary com- 
plex due to a traffic accident. She underwent surgery with consultation through the neurosurgery department at that time. The midface was reconstructed with autologous bone and fixated with metal plates, whereas the supraorbital rim and orbital roof were reconstructed with artificial bone. After that, the reduction site was still vulnerable to sinus-orbit-brain connection after surgery (Fig. 1).

The next day after treatment in the emergency room, the patient came as an outpatient. The cheek swelling still remained with proptosis, and pus was recognized in the conjunctiva. So, we suspected periorbital infection and checked the periorbital area using an enhanced computed tomography (CT). In the CT image, it showed severe sinusitis in the right side as well as a diffuse infectious aspect in the orbit (Fig. 2).

Thus, we planned an emergency operation and conducted a consultation to the otorhinolaryngology department for evaluation of the sinusitis. In the operation room, diffusely spread pus was observed inside the orbit. The bacterial examination was performed in the orbit and in the sinus respectively. Five days later, the same bacteria which Prevotella oralis and methicillin-resistant Staphylococcus epidermidis (MRSA) were identified in both areas.
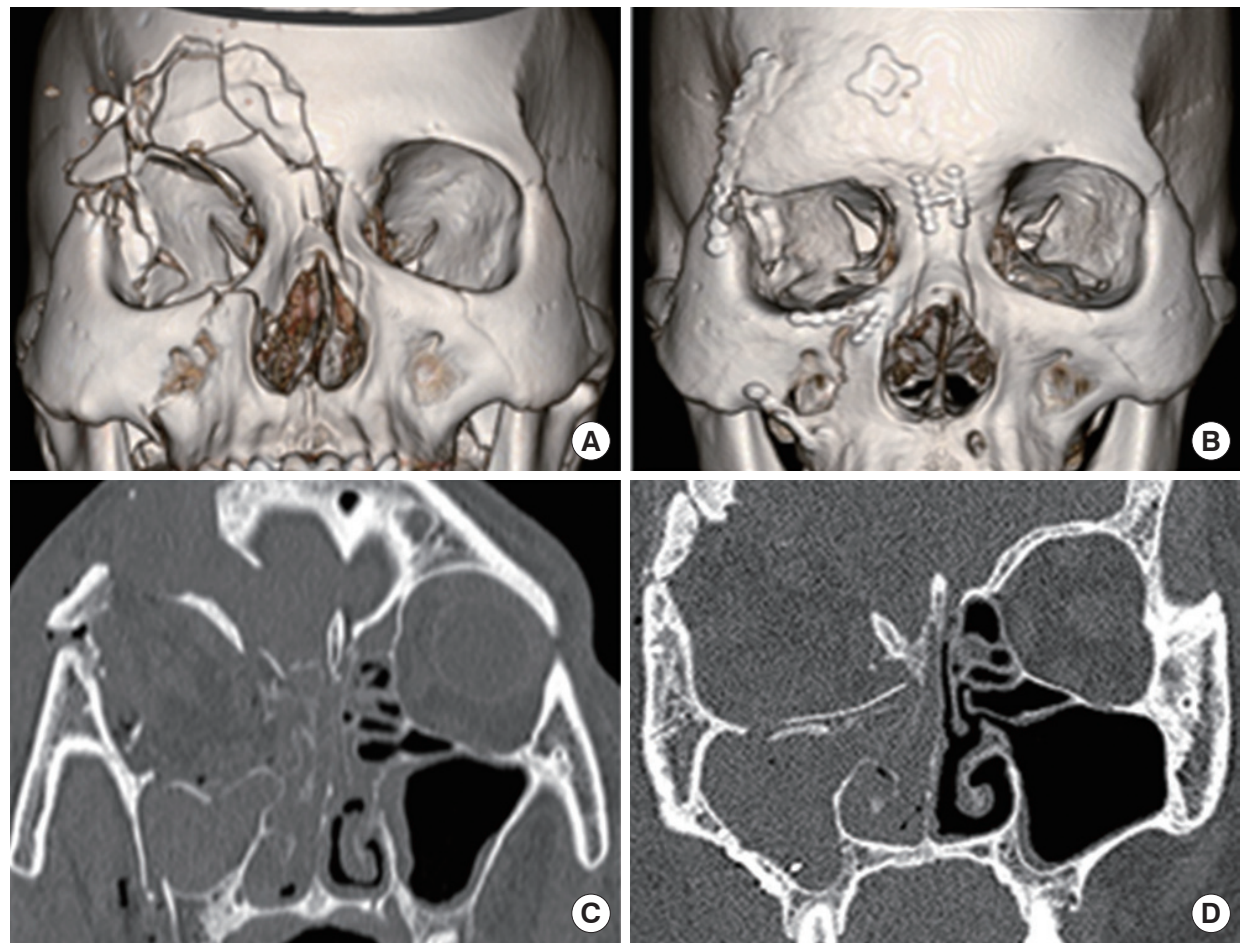

Fig. 1. The patient's computed tomography $(\mathrm{CT})$ scan images in the past. $(\mathrm{A}, \mathrm{C})$ The preoperative CT scan image of the facial bone fracture in 6 years ago. $(\mathrm{B}, \mathrm{D})$ The postoperative CT scan image of the facial bone fracture in 6 years ago.
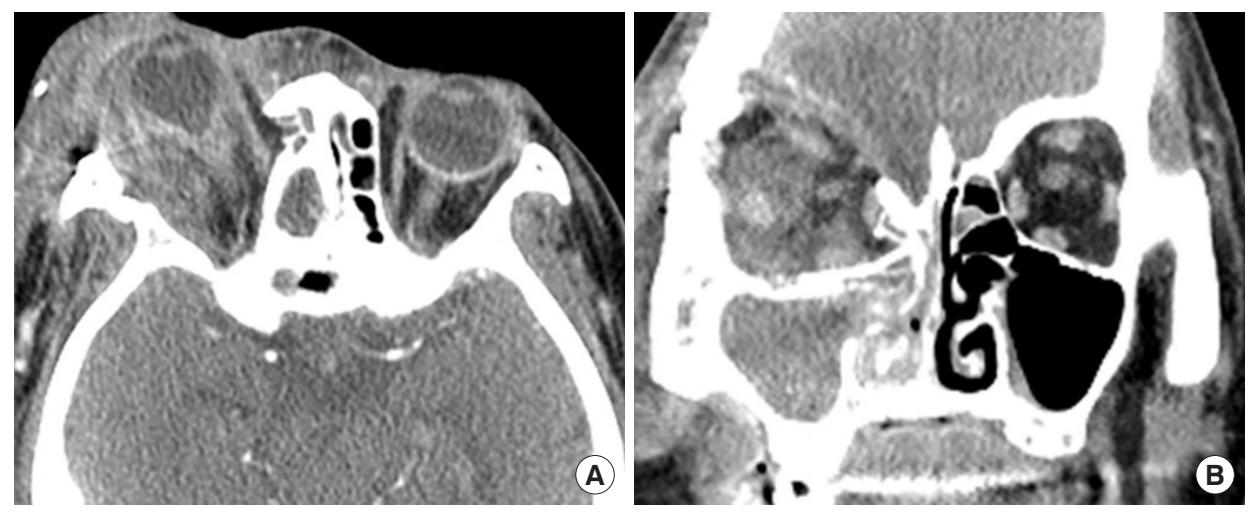

Fig. 2. The patient's enhanced computed tomography (CT) scan images in the present. (A) Diffuse intraorbital infection and proptosis were shown in enhanced CT scan image. (B) Severe sinusitis was shown in enhanced CT scan image. 
Initially, we used experimental intravenous antibiotics such as 3rd generation cephalosporin and aminoglycoside to cover wide spectrum bacteria. But, antibiotics were changed to vancomycin and quinolone after bacterial identification. Medical and surgical treatment, blood test, and radiologic test were regularly performed for more than a month.

As a result, the intraorbital infection was resolved, but blindness and extraocular movement limitation were inevitable (Fig.
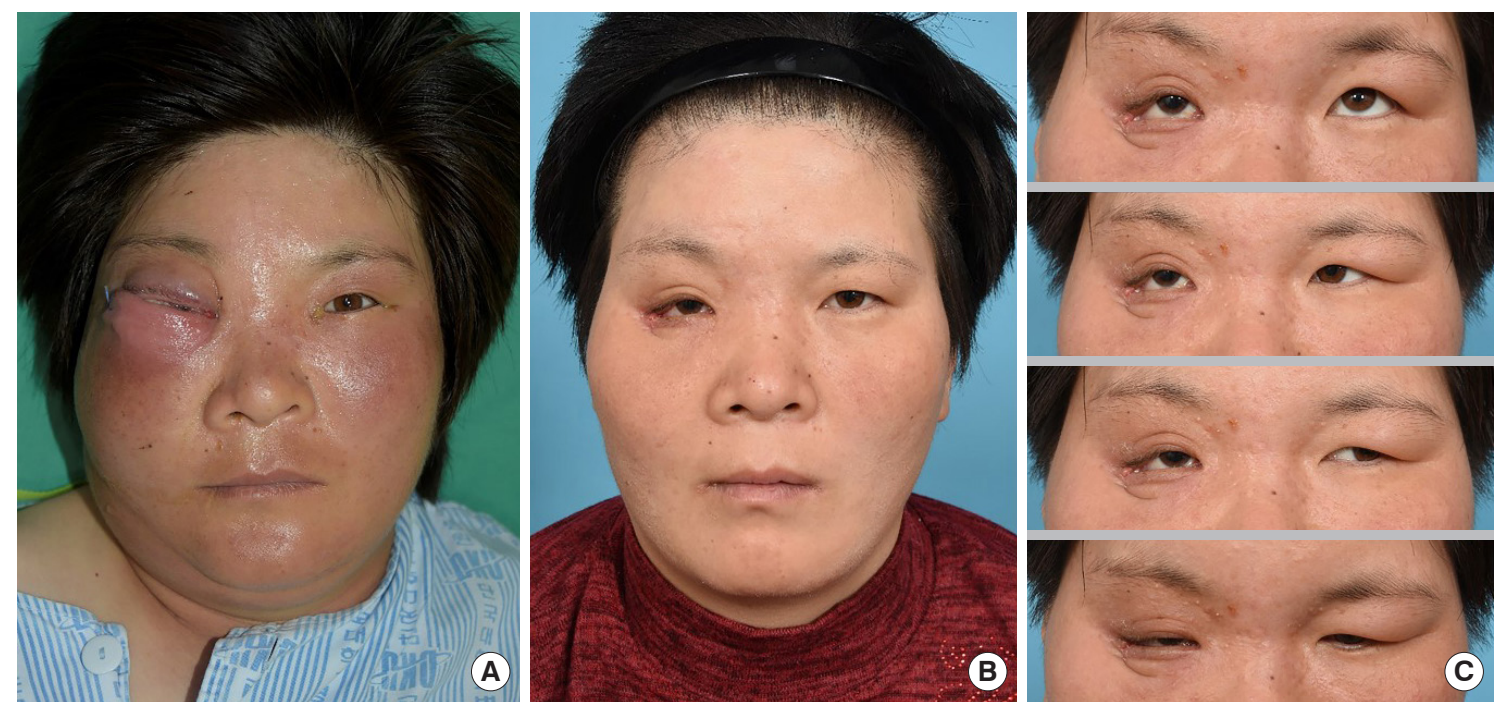

Fig. 3. The patient's clinical photographs. (A) Severe periorbital swelling and proptosis were shown in the initial photograph. (B) Photograph of complete recovery and maintenance 4 months after surgery. (C) Photographs of extraocular movement limitation.
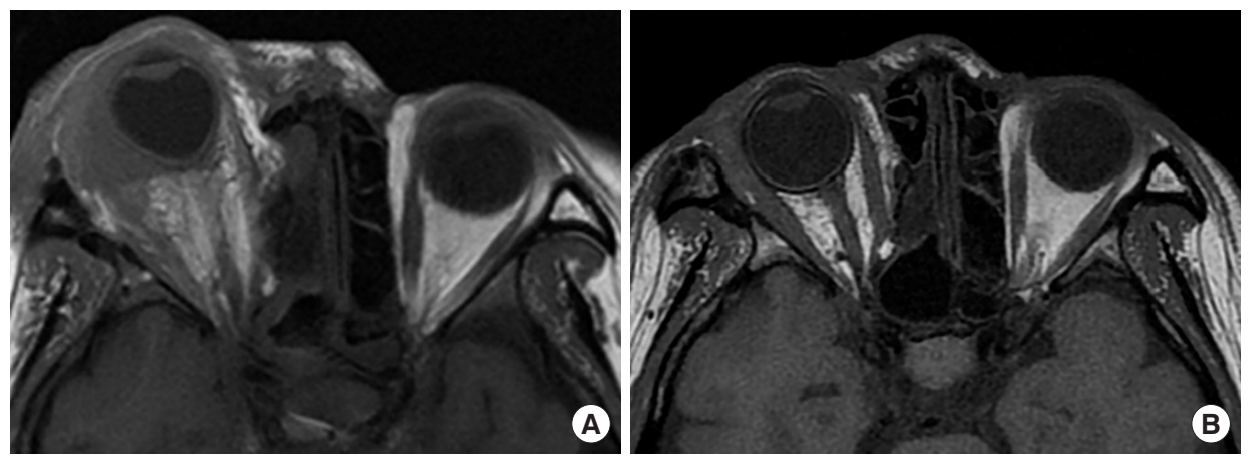

Fig. 4. The patient's magnetic resonance image (MRI). (A) Severe intraorbital infection and proptosis were shown in the initial MRI. (B) Intraorbital infection was totally subsided and maintained in the 4 months later.

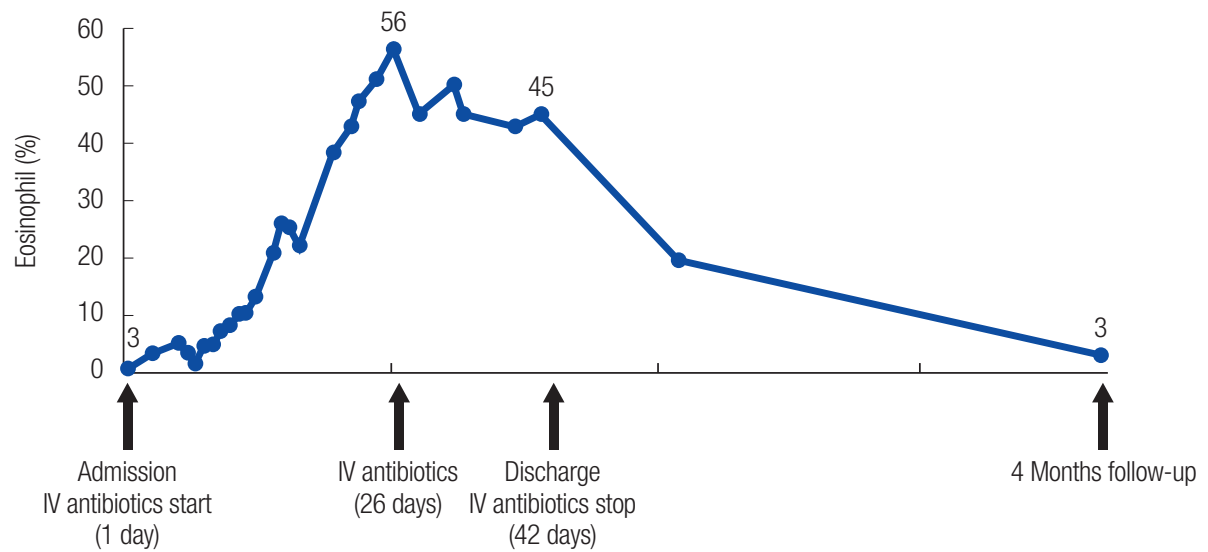

Fig. 5. The eosinophil percentage throughout administration until 4 months after surgery. IV, intravenous. 
3). In radiological evaluation, it revealed right intraorbital diffuse enhancement and paranasal sinusitis in initial magnetic resonance image (MRI) initially, but it showed the improvement in 4-month follow-up MRI image (Fig. 4). One interesting thing was that eosinophilia gradually became worse during the treatment period, but it showed a normal level after improvement (Fig. 5).

\section{DISCUSSION}

Intraorbital infection, which has various etiology and sometimes leaves serious sequelae, has been reported through several articles [1,3-8]. Commonly mentioned in these reports, early diagnosis and active treatment were most important. The diagnosis of orbital infection was made clinically, and imaging helped for confirmation or delineating the source and extension of the infection [1]. Imaging findings were usually correlated to Chandler's clinical classification if the Chandler score was III or higher indicated "significant orbital infection" and needed active treatment [9]. Though incidence rates are low, this type of infection can result in critical situations such as blindness, death or extended infection to the brain [1].

Early-stage infection can be detected by identifying signs of soft tissue swelling, redness and increased warmth of the eyelids. Also, extraocular motility, proptosis, ocular pain, anesthesia of the area innervated by the first and second divisions of the trigeminal nerve, and chemosis can be seen in such infections. A radiological evaluation might help to detect the symptoms of intraorbital infection which the retrobulbar inflammatory changes, extraocular myositis, optic nerve involvement, subperiosteal or orbital abscess, venous engorgement, and cavernous sinus infection or thrombosis using CT or MRI. Besides, the source of infection components could be analyzed through MRI intensity (bacterial infections show low signal centrally on the T1W1 whereas fungal infections show hypointense on T1W1) [2].

Among the many causes of intraorbital infection, paranasal sinusitis is the most common. Trauma, foreign body, skin infection are the second most common, and many other causes exist such as pharyngeal or odontogenic infections, dacryocystitis, ruptured cyst, meningitis, and cavernous sinus thrombosis. The use of implants of foreign material can also cause infection, especially the patients who have immunosuppressive diseases such as diabetes mellitus, septicemia, malignancy, and autoimmune diseases [2]. Our patient had no immunosuppressive condition, but had an opening in the anterior portion of the right maxilla, even after surgery of the facial bone fracture 6 years ago. This communication of the maxillary sinus made the patient more vulnerable to sinus-orbit-brain connection. In this situation, the hematoma caused by trauma triggered the infection to spread from preexisting sinusitis to the orbit and further to the brain. The bacterial identification was confirmed same species both the orbit and sinus such as P. oralis and MRSA, and it supported our hypothesis.

The normal flora of the oral cavity consists of approximately 500 different species. The most common pathogen of intraorbital infection and periodontal disease are, Streptococcus and Staphylococcus spp. respectively. In the younger population, Haemophilus influenzae is a more common cause of intraorbital infection. Tuberculosis and Anaerobic infections (Clostridium or Bacillus) can also cause periorbital infection. Thus, in the case of intraorbital infection, 14-day intravenous vancomycin for MRSA coverage, ceftriaxone for streptococci coverage, and metronidazole for Gram-negative bacilli and anaerobic coverage are recommended [6]. For this case, we used empirical intravenous antibiotics such as 3rd generation cephalosporin and aminoglycoside. After bacterial identification, we used vancomycin and quinolone.

Considering orbital implants' complication rate, the implant itself could also be the cause of infection in our case as well. In Kent et al. [3], 405 cases using hydroxyapatite and porous polyethylene had a complication rate of $9.4 \%$ and $5.6 \%$ respectively for a 10 -year follow-up period. And the hydroxyapatite was reported to cause cellulitis in $0.5 \%$ of a reported case study. In our case, we used titanium plates and porous polyethylene for medial wall and orbital roof reconstruction respectively. The standard treatment would be to extract all implants but extracting the orbital roof implant could cause serious brain damage. This risk posed to be critical to a young female. Thus, we removed all implants without the roof implant after discussed with a neurosurgeon. Fortunately, the infection was successfully controlled without additional complications. Nonetheless, a careful follow up is needed because our patient had a potential risk of infection.

When there is an intraorbital infection, blood tests are also needed regularly. Patients with meningeal sign also should have cerebrospinal fluid tested. In our case, leukocytosis with eosinophilia was identified. We suspect it to be an allergic reaction due to long term injection of antibiotics.

Intraorbital infection can cause various complications ranging from mild to severe. For this reason, early diagnosis and active treatment are essential. To conclude, as presented in our case, close follow up of the maxillary sinus in facial bone fracture patients is important and patients with risk of infection need long-term to follow up for monitoring various infection sources. 


\section{NOTES}

\section{Conflict of interest}

No potential conflict of interest relevant to this article was reported.

\section{Ethical approval}

The study was approved by the Institutional Review Board of Dankook University Hospital (IRB No. 2019-07-018) and performed in accordance with the principles of the Declaration of Helsinki. Written informed consent was obtained.

\section{Patient consent}

The patient provided written informed consent for the publication and the use of her images.

\section{ORCID}

Joo Sung Jung https://orcid.org/0000-0002-2515-9275

Nam Kyu Lim https://orcid.org/0000-0002-0964-983X

Dong Hee Kang https://orcid.org/0000-0002-6673-9655

\section{REFERENCES}

1. Abdouramani O, Nguefack S, Dohvoma V, Moifo B, Eballe AO, Moho A, et al. Bilateral intraorbital abscesses with intracranial complications in a young Cameroonian girl: a case re- port. Clin Ophthalmol 2012;6:1429-32.

2. Hershey BL, Roth TC. Orbital infections. Semin Ultrasound CT MR 1997;18:448-59.

3. Kent SS, Kent JS, Allen LH. Porous polyethylene implant associated with orbital cellulitis and intraorbital abscess. Can J Ophthalmol 2012;47:e38-9.

4. Kim YH, Kim H, Yoon ES. Unrecognized intraorbital wooden foreign body. Arch Craniofac Surg 2018;19:300-3.

5. Udaondo P, Garcia-Delpech S, Diaz-Llopis M, Salom D, Garcia-Pous M, Strottmann JM. Bilateral intraorbital abscesses and cavernous sinus thromboses secondary to Streptococcus milleri with a favorable outcome. Ophthalmic Plast Reconstr Surg 2008;24:408-10.

6. Vu Q, Dyer G, Kunjukunju N. A rare case of odontogenic parainfectious intraorbital optic neuritis with the absence of clinical manifestation of orbital cellulitis. Retin Cases Brief Rep 2015;9:205-9.

7. Boonsopon S, Tesavibul N, Uiprasertkul M, Leeamornsiri S, Choopong P. Rare presentation of intractable tuberculous panophthalmitis with intraocular and intraorbital abscesses: a case report. J Med Case Rep 2017;11:180.

8. Zafar MA, Waheed SS, Enam SA. Orbital aspergillus infection mimicking a tumour: a case report. Cases J 2009;2:7860.

9. Rudloe TF, Harper MB, Prabhu SP, Rahbar R, Vanderveen D, Kimia AA. Acute periorbital infections: who needs emergent imaging? Pediatrics 2010;125:e719-26. 